\title{
44005 - PREOPERATIVE CATABOLISM AND THE ANABOLIC EFFECTS OF NUTRITION
}

\author{
Ralph Lattermann, Department of Anesthesia, Royal Victoria Hospital, McGill, Montreal, \\ QC, Canada; \\ Linda Wykes, School of Dietetics and Human Nutrition, McGill University; \\ Evan Detschman, School of Dietetics and Human Nutrition, McGill University; \\ L Eberhart, Department of Anesthesia and Intensive Care, Marburg University; \\ $S$ Meterissian, Department of Surgery, RVH, McGill University; \\ T Schricker, Department of Anesthesia, RVH, McGill University;
}

INTRODUCTION: The goal of the present study was to investigate whether the anticatabolic effects of hypocaloric nutrition after colorectal surgery depends on the patients` preoperative catabolic state, the hypothesis being that catabolic patients benefit particularly from perioperative nutritional support.

METHODS: After approval by the local Ethics Committee and after obtaining written informed consent, seventeen metabolically healthy patients undergoing colorectal surgery for cancer were studied. The patients' catabolic state was assessed by measuring whole body protein balance using primed continuous infusions of L-[1-13C]leucine before and two days after surgery, with positive values indicating anabolism. All patients received intravenous glucose with amino acids over 72h starting at 12:00 on the day before surgery until the second postoperative day. Glucose provided $50 \%$ of the patients' resting energy expenditure (REE). Amino acids were infused at rates providing $20 \%$ of the REE. Body mass index (BMI) and preoperative weight loss were recorded. Circulating concentrations of albumin, insulin and cortisol also were determined. The association between the pre- and the postoperative protein balance was assessed using the Spearman rank correlation test. Other factors potentially associated with the anabolic response to perioperative nutrition including sex, age, body mass index, weight loss, duration of surgery, perioperative blood loss, circulating albumin, insulin and cortisol concentrations were also analyzed in a univariate model (ANOVA for continuous variables and the Wilcoxon's U-test for dichotomous variables). Variables that were somewhat associated with postoperative protein balance (defined as a p-value $\& \# 8804 ; 0.2$ ) were subjected to a stepwise backward linear regression analysis.

RESULTS: A highly significant inverse correlation between preoperative protein balance and the anabolic response to nutrition was observed $(\mathrm{p}<0.0001)$, i.e. patients with a higher degree of preoperative protein catabolism had a more pronounced increase in postoperative protein balance $\left(r^{2}=0.73\right.$, Spearman correlation coefficient $\left.=0.85\right)$.

All variables with potential relevance for postoperative protein balance, except age were removed as not significant during the stepwise linear regression procedure, leaving the preoperative protein balance and patient's age as the only two factors in the model. The $\mathrm{r}^{2}$ for this model is 0.85 with a root mean square error (RMSE) of 2.77. The equation of the final regression model is: 
Protein balancepostOP $=19.1-0.20 \mathrm{x}$ age [years] $+0.58 \times$ protein balancepreOP DISCUSSION: The anabolic effect of hypocaloric nutrition after colorectal surgery is more pronounced in elderly patients and in patients with a higher degree of preoperative protein catabolism. Other indicators of catabolism, i.e. circulating albumin concentrations or BMI do not affect the protein-sparing effect of hypocaloric nutrition in surgical patients. 